\title{
Endocarditis Infecciosa en Hospital de Talca, período 1998 - 2015
}

\author{
Jaime Cruz ${ }^{1}$, Patricio Marín ${ }^{2}$, Daniela Migueles ${ }^{1}$ \\ 1. Servicio de Medicina, Hospital Regional de Talca, Talca, Chile \\ 2. Servicio de Cardiología, Hospital Regional de Talca, Talca, Chile
}

Introducción: La endocarditis infecciosa es una enfermedad con una alta mortalidad que se ha mantenido estable en los últimos años pese a avances en su diagnóstico y tratamiento.

Objetivo: Evaluar las características clínicas de los pacientes con Endocarditis Infecciosa tratados en el Hospital de Talca.

Materiales y métodos: Estudio retrospectivo en que se revisaron las historias clínicas de los pacientes con endocarditis infecciosa atendidos entre 1998 y 2015 en el Hospital Regional de Talca.

Resultados: Se encontraron 62 pacientes con endocarditis infecciosa con un promedio de edad de 49,7 años, $61,2 \%$ de ellos de sexo masculino, $21 \%$ de ellos con válvula protésica y $13 \%$ con antecedentes de enfermedad reumática. Las comorbilidades más frecuentes fueron hipertensión arterial, diabetes mellitus y enfermedad renal crónica. El 72,5\% de los pacientes se presentaron con síndrome febril y soplo asociado. Un $87,1 \%$ presentaban vegetaciones al ecocardiograma. El $67,1 \%$ de los pacientes tenía hemocultivo positivo, de los cuales el microorganismo más frecuente fue el Staphylococcus aureus (22,6\%). Un 24,2 \% de los pacientes requirieron tratamiento quirúrgico. La mortalidad intrahospitalaria fue de un $19,3 \%$.

Conclusiones: Las características clínicas de los pacientes descritos son similares a estudios nacionales e internacionales, con una mortalidad intrahospitalaria elevada.

Palabras Claves: Endocarditis, infecciosa; Mortalidad intrahospitalaria; Staphylococcus aureus.

Correspondencia:

Jaime Cruz

jcruzfuentes@gmail.com 
Background: Infective endocarditis is a disease with high mortality that remains stable in recent years despite advances in it's diagnosis and treatment.

Aim: Evaluate the clinical characteristics of patients with infective endocarditis treated at Hospital de Talca, Chile.

Material and Methods: Retrospective study in which the clinical records of patients with infective endocarditis treated between 1998 and 2015 at Hospital Regional de Talca were reviewed.

Results: 62 patients with infective endocarditis were identified. The median age was 49,7 years, $61,2 \%$ were males. $21 \%$ carried one or more prosthetic heart valve and $13 \%$ had previous rheumatic valve disease. The most common comorbidities were hypertension, diabetes, and chronic kidney disease. $72,5 \%$ presented with fever and a cardiac murmur, $87,1 \%$ had vegetations in echocardiography. In $67 \%$ of patients blood cultures were positive and22,6\% of them had blood cultures positive for Staphylococcus aureus. $24,2 \%$ of patients required hearth surgery. Hospital mortality was $19,3 \%$. Conclusions: The clinical characteristics of patients included in this series were similar to those found in national and international studies, Hospital mortality was high

Key words: Endocarditis, infective; Hospital mortality; Staphylococcus aureus 


\section{Introducción:}

La endocarditis infecciosa (EI) es una enfermedad grave, compleja en su diagnóstico y manejo por lo que requiere ser tratada por equipos multidisciplinarios ${ }^{1}$. Tiene una incidencia de aproximadamente 1,7 a 7,9 casos por cada 100.000 habitantes al año ${ }^{1,2}$; en Chile, se estima que sería de 2 casos por 100.000 pacientes por año en el área central de Santiago ${ }^{3}$. Pese a los avances en el tratamiento, la endocarditis está asociada a una mortalidad intrahospitalaria que se ha mantenido estable en un 15 a $20 \%$ durante los últimos 25 años según series internacionales ${ }^{4,5,6}$. Además, la EI se asocia a mal pronóstico a largo plazo, con una mortalidad que al año de diagnóstico alcanza un $40 \%{ }^{4}$.

La dificultad para disminuir la mortalidad puede ser explicada por la constante evolución epidemiológica de esta enfermedad $^{1,2,7}$. Clásicamente, la endocarditis se producía en pacientes susceptibles con valvulopatía causada por enfermedad reumática ${ }^{2,7,8,9}$ y el Streptococcus viridans era el patógeno más frecuente ${ }^{7,8,9}$. Actualmente, ha disminuido la proporción de pacientes con antecedentes de enfermedad reumática o con hallazgos ecocardiográficos sugerentes ${ }^{10} \mathrm{y}$ el microorganismo más frecuentemente encontrado es el Staphylococcus aureus, comprometiendo a más del $25 \%$ de los pacientes con $\mathrm{EI}^{1}{ }^{1,}, 10,11$ lo que podría estar en relación con el aumento de individuos con válvulas protésicas, inmunodeprimidos, en hemodiálisis y usuarios de drogas endovenosas ${ }^{12}$. El hecho de que el Staphylococcus aureus sea el microorganismo más frecuente en causar EI es importante en la evolución de los pacientes debido a que le confiere un peor pronóstico asociado a mayor frecuencia de complicaciones y mortalidad intrahospitalaria que las endocarditis causadas por otros microorganismos. ${ }^{6,10,13}$

Este estudio tiene como propósito describir la realidad de nuestro centro hospitalario y determinar si los pacientes atendidos con endocarditis infecciosa presentan similares características a los de estudios nacionales e internacionales.

\section{Material y método}

Se realizó un estudio descriptivo, mediante la búsqueda de todos los pacientes mayores de 15 años con el diagnóstico de Endocarditis infecciosa basados en criterios de Duke y Duke modificado ${ }^{14}$ atendidos en el Hospital Regional de Talca entre el 1 de enero de 1998 y 31 de diciembre de 2015 en los registros de los Servicios de Cardiología y Medicina del Hospital. Además, se usó la base de datos del Sistema Informático de la Región del Maule (SisMaule). Se revisaron en forma retrospectiva las historias clínicas y epicrisis de los pacientes seleccionados para el estudio y se consignaron los datos demográficos y clínicos pertinentes.
Los datos fueron registrados en planilla Microsoft Excel 2016®, el análisis descriptivo se realizó utilizando promedio y desviación estándar en variables cuantitativas y porcentajes en variables cualitativas.

\section{Resultados}

Se encontraron en los registros del Hospital Regional de Talca y SisMaule desde 1998 al 2015 a 77 pacientes mayores de 15 años en cuyo ingreso o egreso hospitalario se registró el diagnóstico de Endocarditis infecciosa. Finalmente, se analizaron en este estudio 62 pacientes que cumplían Criterios de Duke o Duke modificado ${ }^{14}$, se excluyeron 15 pacientes, debido a que 9 no tenían ficha clínica disponible o datos suficientes para establecer el diagnóstico y 6 presentaban un diagnóstico alternativo a endocarditis. El $19,4 \%$ de los casos (12 pacientes) fueron diagnosticados en los últimos años de revisión.

\begin{tabular}{|c|c|c|}
\hline \multicolumn{3}{|c|}{$\begin{array}{l}\text { Tabla 1: Cardiopatías y comorbilidades previas al diagnóstico } \\
\text { endocarditis }\end{array}$} \\
\hline Cardiopatías & $\mathrm{n}$ & $\%$ \\
\hline Prótesis valvular & 13 & 21,0 \\
\hline Mecánica & 8 & 13,0 \\
\hline Biológica & 5 & 8,1 \\
\hline Enfermedad Reumática & 8 & 13,0 \\
\hline Cardiopatía Congénita & 6 & 9,7 \\
\hline Cardiopatía Isquémica & 3 & 4,8 \\
\hline Marcapaso & 1 & 1,6 \\
\hline \multicolumn{3}{|l|}{ Comorbilidades } \\
\hline Hipertensión arterial & 17 & 27,0 \\
\hline Diabetes Mellitus & 15 & 24,0 \\
\hline Insuficiencia Renal & 7 & 11,3 \\
\hline Alcoholismo & 2 & 3,2 \\
\hline Uso de inmunosupresores & 2 & 3,2 \\
\hline Embarazo & 1 & 1,6 \\
\hline Hemodiálisis & 1 & 1,6 \\
\hline
\end{tabular}

La edad promedio fue 49,7 $\pm 15,7$ años; un $61,2 \%$ (38) de los pacientes eran de sexo masculino. En la Tabla 1 se presentan las cardiopatías subyacentes y comorbilidades encontradas en los pacientes. Las cardiopatías subyacentes más frecuentes fueron la prótesis valvular $(21 \%)$ enfermedad Reumática (13\%) y cardiopatía congénita $(9,7 \%)$. Las comorbilidades más prevalentes en el grupo estudiado fueron hipertensión arterial (27\%), diabetes mellitus (24\%) y enfermedad renal crónica $(11,29 \%)$, con sólo 1 paciente sometido a hemodiálisis. 
En cuanto a la forma de presentación de la endocarditis, un $72,5 \%$ se manifestó como síndrome febril prolongado asociado a soplo cardiaco, un 40,3\% presentó insuficiencia cardiaca al ingreso; $22,5 \%$ insuficiencia renal aguda; $12,9 \%$ un síndrome inmunológico asociado a endocarditis y $17,7 \%$ embolia (10 fueron cerebrales y 1 esplénica).

Respecto al uso de ecocardiograma, a 57 pacientes se les realizó ecocardiograma transtorácico y a 36 transesofágico. Las lesiones encontradas fueron vegetaciones $(87,1 \%)$ en 554 casos; abscesos en $3(4,8 \%)$ y 1 caso con rotura valvular $(1,6 \%)$. La válvula más afectada por endocarditis fue la aórtica $(50,0 \%)$, luego mitral $(27,4 \%)$, mitro aórtica $(9,7 \%)$, tricúspide $(6,5 \%)$ y pulmonar $(1,6 \%)$. En al caso de las endocarditis por válvula protésica, de los 13 pacientes, 5 eran biológicas ( 2 mitrales, 1 aórtica y 2 mitro aórticas) y 8 mecánicas (4 mitrales, 3 aórticas y 1 mitro aórtica).

Se logró aislar el microorganismo causante mediante hemocultivo en 42 casos $(67,7 \%)$. 14 resultaron negativos sin antibióticos previos $(22,6 \%)$ y 5 con antibióticos $(8,1 \%)$. Sólo en 1 caso no fue posible encontrar registro del cultivo. En la Tabla 2 se presentan los microorganismos encontrados según informes de laboratorio. El microorganismo aislado con más frecuencia resultó ser el Staphylococcus aureus en un 22,6\% de los casos. En las EI asociadas a válvulas protésicas se logró aislar un microorganismo en el 69,2\% de los casos, siendo el más frecuente el Stahylococcus coagulasa negativo $(23,0 \%)$, luego el Staphylococcus aureus y Enterococcus faecalis (ambos con 15,4\%).

\begin{tabular}{|l|c|c|}
\hline \multicolumn{3}{|l|}{ Tabla 2: Microorganismos aislados en Hemocultivos } \\
\hline Microorganismo & n & $\%$ \\
\hline Staphylococcus aureus & 14 & $22,6 \%$ \\
\hline Staphylococcus coagulasa negativo & 9 & $14,5 \%$ \\
\hline Streptococcus sp & 6 & $9,7 \%$ \\
\hline Enterococcus faecalis & 2 & $3,2 \%$ \\
\hline Streptococcus viridans & 2 & $3,2 \%$ \\
\hline Streptococcus agalactiae & 2 & $3,2 \%$ \\
\hline Corynebacterium sp & 1 & $1,6 \%$ \\
\hline Acinetobacter baumannii & 1 & $1,6 \%$ \\
\hline Bacilo gram positivo difteromorfo & 1 & $1,6 \%$ \\
\hline Bacilo gram negativo & 1 & $1,6 \%$ \\
\hline Streptococcus pneumoniae & 1 & $1,6 \%$ \\
\hline Pseudomonas aeruginosa & 1 & $1,6 \%$ \\
\hline Enterococcus sp & 1 & $1,6 \%$ \\
\hline Sin cultivo o negativo & 20 & $32,3 \%$ \\
\hline
\end{tabular}

\begin{tabular}{|l|c|c|}
\hline $\begin{array}{l}\text { Tabla 3: Indicaciones de Resolución quirúrgica en los pacientes } \\
\text { sometidos a cirugía }\end{array}$ \\
\hline Indicación Cirugía & $\mathbf{n}$ & $\%$ \\
\hline Insuficiencia Cardiaca & 11 & 73,3 \\
\hline Sepsis no controlada & 4 & 26,7 \\
\hline Disfunción valvular & 4 & 26,7 \\
\hline Vegetaciones sobre 10 mm & 3 & 20,0 \\
\hline Absceso & 2 & 13,3 \\
\hline Embolias múltiples & 1 & 6,7 \\
\hline
\end{tabular}

Se efectuó tratamiento quirúrgico en el $24,2 \%$ de los pacientes. En la Tabla 3 se resumen las indicaciones de cirugía. La más frecuente fue la insuficiencia cardiaca, luego la sepsis no controlada y finalmente la disfunción valvular. Dos pacientes con EI y válvula protésica requirieron cirugía $(15,4 \%)$ siendo la insuficiencia cardiaca la indicación en ambos casos.

La mortalidad intrahospitalaria fue 19,4\% (12 pacientes). En los pacientes con EI por válvula protésica se observó una mortalidad de 15,3\% (2 de 13 pacientes).

\section{Discusión}

Nuestro hospital es el centro de referencia para la atención de los pacientes de la Región del Maule Sin embargo, al no contar con cirugía cardiaca durante el período en el cual se realizó este estudio no es posible asegurar que se hayan atendido a todos los pacientes de la región con endocarditis infecciosa, ya que aquellos con indicación quirúrgica atendidos en hospitales de menor complejidad pudieron ser derivados directamente a los centros que contaban con ella.

Destaca el aumento del diagnóstico de EI en los últimos dos años del estudio, lo que podría indicar un aumento de la prevalencia de endocarditis en nuestra región o un subdiagnóstico de endocarditis en los años previos.

La edad de nuestros pacientes estudiados es en prome-

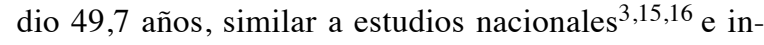
ternacionales ${ }^{8,12}$, También es similar la mayor afección de pacientes de sexo masculino con una razón aproximada a una razón 2:1 respecto de las mujeres. ${ }^{3,8,15,16}$

En nuestro estudio existió una mayor proporción de pacientes con endocarditis en válvulas protésicas que en otros estudios nacionales ${ }^{3,15}$, pero es similar a la proporcionada por estudios internacionales ${ }^{17}$ en los cuales se encuentra en un $20 \%$ de los pacientes. Esto es relevante debido a que la EI por válvula protésica tiene asociado un mayor riesgo de mortalidad ${ }^{13}$. Destaca 
también que los pacientes ingresados a nuestro estudio tenían menos antecedentes de enfermedad reumática que en estudio nacionales de mayor antigüedad 3,16 pero es similar a la descrita en el estudio de Stockins ${ }^{15}$ realizado en el hospital de Temuco entre el año 2003 y 2010. Este hecho podría explicarse por la tendencia a la baja de la incidencia de esta enfermedad a nivel global..$^{3,9}$

Las comorbilidades más frecuentes de nuestros pacientes fueron la hipertensión, diabetes mellitus y enfermedad renal crónica. Los 2 últimas son factores de riesgo para el desarrollo de endocarditis y mortalidad intrahospitalaria $6,{ }^{13,15,18}$, especialmente en los pacientes que requieren administración de insulina. $\mathrm{N}$ está claramente definido el valor pronóstico del control estricto de la glicemia en los pacientes con endocarditis y, por lo tanto, es ideal mantener el tratamiento en forma similar al de otros pacientes críticos.

La forma de presentación fue similar a estudios nacionales $^{15,16}$, siendo más frecuente el hallazgo de soplo en un paciente cursando con síndrome febril que en nuestra serie representó un 72,5\% de los casos. La insuficiencia cardiaca estaba presente al ingreso en $40 \%$ de nuestros pacientes, varios de los cuales requirieron finalmente intervención quirúrgica.

El hallazgo más frecuente en el ecocardiograma fue la vegetación, raramente se encontraron abscesos y rotura valvular. En los pacientes que presentaron estas últimas dos complicaciones fue necesaria la cirugía. La válvula más afectada fue la aórtica y en segundo lugar la mitral, hallazgos compartidos con otros estudios nacionales $^{3,15}$. Con baja frecuencia se vieron afectadas las válvulas del lado derecho del corazón.

Destaca una similar proporción de hemocultivos negativos respecto a estudios nacionales ${ }^{3,15,16}$, resultados que pueden ser en parte debido al uso de cultivos automatizados. Una proporción importante de estos cultivos negativos fueron causados por el uso de antibióticos antes de la toma del cultivo lo que resulta un problema común en la práctica y que está asociada a retraso del diagnóstico y probabilidad de tener una falta de respuesta en el tratamiento antibiótico ${ }^{19}$. En los hemocultivos que resultaron positivos, como es la tendencia actual, el microorganismo aislado más frecuente fue el Staphylococcus aureus ${ }^{4,9}$, aunque por el amplio periodo de estudio (17 años) su frecuencia pudiera estar subestimada. Sin embargo, en los últimos 2 años de estudio no se observó una mayor tendencia a presentar EI por Staphylococcus aureus.

La mortalidad intrahospitalaria fue menor a la de otros estudios nacionales ${ }^{3,15}$, pero manteniendo la tendencia entre 15 a $20 \%$ 4,5,6. Este valor puede estar subestimado debido a que al no contar con Cirugía cardiaca en nuestro centro durante el período en el que se realizó este estudio, se derivaban a otros centros para la intervención quirúrgica y en algunos pacientes no se encontraron los registros post derivación.

Respecto a la cirugía, existen registros de hospitales como el nuestro sin acceso a cirugía cardiaca, en donde se determinó que la mortalidad no resulta significativamente relacionada al centro hospitalario en donde se atiende el paciente ${ }^{20}$, sino que se relaciona más con la persistencia de la sepsis, las complicaciones cardiacas y la edad del paciente ${ }^{6,20}$. A partir de estos datos se concluye que no es una limitante tratar a los pacientes en un centro sin cirugía cardiaca, pero es importante derivar en forma oportuna a los pacientes que desarrollen complicaciones que requieran intervención quirúrgica, disminuyendo el riesgo de mortalidad en el episodio agudo $^{15}$. Pese a lo descrito, es preocupante que solo un $24,2 \%$ de nuestros pacientes fueran derivados para tratamiento quirúrgico, ya que es un número mucho menor al de series nacionales similares en las cuales el tratamiento quirúrgico va desde un 35,3 a 52\% 3,15,16. Eso sugiere que no estamos realizando una derivación oportuna de pacientes con indicación quirúrgica.

Algunas limitantes de este estudio, además de las inherentes a ser un estudio observacional retrospectivo, son la pérdida de datos producto del registro manual y la ausencia de registros electrónicos en los pacientes más antiguos.

En conclusión, las características de nuestros pacientes están acordes a los cambios demográficos y epidemiológicos de la endocarditis infecciosa en los últimos años. 


\section{Referencias}

1) FERNÁNDEZ-HIDALGO N, ALMIRANTE B. La endocarditis infecciosa en el siglo XXI: cambios epidemiológicos, terapéuticos y pronósticos. Enferm Infecc Microbiol Clin 2012; 30: 394-406.

2) PRENDERGAST BD. The Changing face of infective endocarditis. Heart 2006; 92: 879-85.

3) OYONARTE M, MONTAGNA R, BRAUN S, ROJO P, JARA J, CERECEDA M, et al. Endocarditis infecciosa: características clínicas, complicaciones y mortalidad en 506 pacientes y factores pronósticos de sobrevida a 10 años (1998-2008). Estudio cooperativo nacional en endocarditis infecciosa en Chile (ECNEI-2). Rev Med Chile 2012; 140: 1517-1528.

4) MURDOCH D, COREY R, HOEN B, MIRÓ J, FOWLER V, BAYER AS, et al. Clinical Presentation, Etiology and Outcome of Infective Endocarditis in the 21st Century: The International Collaboration on Endocarditis - Prospective Cohort Study. Arch Intern Med 2009, 169: 463-73.

5) WANG A. The Changing Epidemiology of Infective Endocarditis. JACC 2012; 59: 1977-8.

6) CHU V, CABELl C, BENJAMIN D, KUNIHOLM E, FOWLER JR V, ENGEMANN J, et al. Early Predictors of In Hospital Death In Infective Endocarditis. Circulation 2004; 109: 1745-9.

7) BASHORE T, CABELL C, FOWLER JR V. Update on Infective Endocarditis. Current Problems in Cardiology 2006; 31: 274-352.

8) CASTILLO J, ANGUITA M, RUIZ M, PEÑA L, SANTISTEBAN M, PUENTES M, et al. Cambios epidemiológicos de la endocarditis infecciosa sobre válvula nativa. Rev Esp Cardiol 2010; 64: 594-8.

9) CABELL C, JOLLIS C, PATERSON G, COREY G, ANDERSON D, SEXTON DJ, et al. Changing Patient Characteristics and the effect on Mortality in Endocarditis. Arch Intern Med 2002; 162: 90-4.

10) SELTON-SUTY C, CELARD M, LE MOING V, DOCO-LECOMPTE T, CHIROUZE C, IUNG B, et al. Preeminence of Staphylococcus aureus in Infective Endocarditis: a 1-year po- pulation-based survey. Clinical Infectous Diseases 2012; 54: 1230-9.

11) HOEN B, DUVAL $X$. Infective Endocarditis. N Eng J Med 2013; 368: 1425-33.

12) HERMIDA A, LÓPEZ R, RODRÍGUEZ M, LADO F. Estudio retrospectivo de la endocarditis infecciosa en diferentes grupos de riesgo. Rev Med Chile 2007; 135: 11-6.

13) DELAHAYE F, ALLA F, BEGUINOT I, BRUNEVAL P, DOCO-LECOMPTE T, LACASSIN F, et al. In-hospital mortality of infective endocarditis: Prognostic factors and evolution over an 8-year period. Scandinavian Joirnal of Infectious Diseases 2007; 39: 849-57.

14) LI JS, SEXTON DJ, MICK N, NETTLES R, FOWLER V, RYAN T, et al. Proposed Modifications to the Duke Criteria for the diagnosis of infective endocarditis. Clin Infect Dis 2000; 30: 633-8.

15) STOCKINS B, NEIRA V, PAREDES A, CASTILLO C, TRONCOSO A. Perfil clínico-epidemiológico de pacientes con endocarditis infecciosa período 2003-2010 en el hospital de Temuco, Chile. Rev Med Chile 2012; 140: 1304-1311.

16) BRAUN S, ESCALONA P, CHAMORRO S, CORBALAN H, PEREZ C, LABARCA J, et al. Endocarditis Infecciosa: análisis de 261 casos y resultados del tratamiento en un enfoque multidisciplinario. Rev Med Chile 2000; 128: 708-20.

17) WANG A, ATHAN E, PAPPAS P, FOWLER JR V, OLAISON L, PARÉ C, et al. Contemporary Clinical Profile and Outcome of Prosthetic Valve Endocarditis. JAMA 2007; 297: 1354-61.

18) WALLACE S, WALTON B, KHARBANDA R, HARDY R, WILSON A, SWANTON R. Mortality from infective endocarditis: clinical predictor of outcome. Heart 2002; 88: 53-60.

19) CASABÉ J. Endocarditis Infecciosa. Una Enfermedad Cambiante. Medicina (B.Aires) 2008; 68: 164-74.

20) RODRÍGUEZ-ESTEBAN M, ANGUITA M, MIRANDA-MONTERO S, CASTILLO J, ÁLVAREZ-COSTA L, LLORENS-LEÓN R, et al. Endocarditis infecciosa en 2 hospitales universitarios españoles que difieren en su localización y en la presencia de servicio quirúrgico. Enferm Infecc Microbiol Clin 2014; 32: 297-301 